Supporting Information for:

\title{
Novel ferroelectric and antiferroelectric smectic and columnar mesophases in fluorinated symmetrical bent-core compounds
}

\author{
R. Amaranatha Reddy, V. A. Raghunathan and B. K. Sadashiva \\ Raman Research Institute, C.V. Raman Avenue, Sadashivanagar \\ Bangalore 560 080, India.
}

(1) Figure

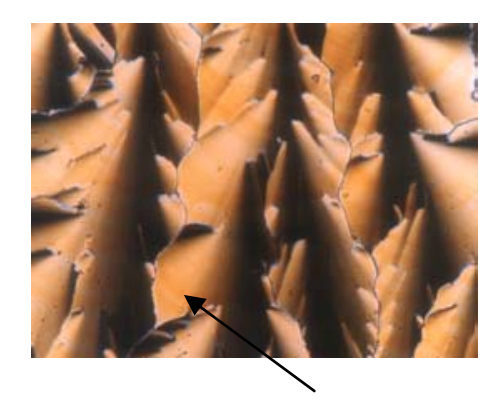

Figure S1. Optical photomicrograph obtained in the $\mathrm{Col}_{\mathrm{rF}}$ phase of compound 17. The sharp yellow lines over the focal-conic texture (shown by arrow) gradually appear at lower temperature. 


\section{(2) Experimental Section}

\section{(a) Measurements}

The textural observations were made under an optical polarized light microscope (Leitz Laborlux 12 POL/ Olympus BX 50) connected to a heating stage and a central processor (Mettler, Models FP 82 HT and FP 90 respectively). A differential scanning calorimeter (Perkin-Elmer, Model Pyris 1D) was used to determine the transition temperatures and the associated enthalpies of the mesophases under investigation. The structure of the mesophases obtained were determined by XRD measurements using a $4 \mathrm{~kW}$ rotating anode X-ray source (Rigaku Ultrax 18) with graphite crystal monochromated $\mathrm{Cu}-\mathrm{K}_{\alpha}$ radiation $(1.54 \AA)$. The diffraction patterns of the unoriented samples were collected on an imaging plate (Marresearch). The polarization measurements were carried out using a triangular-wave method. The triangular-wave voltages were generated from a waveform generator (Wavetek, Model 39), which was amplified using TREK MODEL 601B-3 amplifier. The current response traces were recorded using a Tektronix Oscilloscope, TDS 220. The dc field switching characteristics were also carried out for some of the compounds using a Regulated Dual DC Power Supply (APLAB, Model LD6401).

In order to confirm the chemical structure of the compounds synthesized, NMR $\left({ }^{1} \mathrm{H}\right.$ and ${ }^{13} \mathrm{C}$ ) spectra were recorded using a Bruker AMX 400 spectrometer (tetramethylsilane as an internal reference) and the infrared spectra were obtained on a Shimadzu FT IR-8400 spectrophotometer. The elemental analysis was carried out using a Carlo-Erba 1106 analyzer. All the target materials as well as the intermediate compounds were purified by column chromatography (silica gel, 60-120 mesh) and were crystallized using suitable analytical grade solvents. The purity of all these compounds was confirmed using normal 
phase high performance liquid chromatography ( $\mu$-Porasil column, $3.9 \mathrm{~mm} \times 300 \mathrm{~mm}$, Waters Associates Inc.) and using 1\% acetone in dichloromethane as the eluent.

\section{(b) Synthetic Procedure and Analytical data}

The detailed synthetic procedure as well as the analytical data for all the compounds of series II is given below.

2,7-Naphthylene bis (4-benzyloxybenzoate), (3, synthetic scheme)

A mixture of 2,7-dihydroxynaphthalene (1) (1.6g, $10 \mathrm{mmol})$, 4-benzyloxybenzoic acid (2) $(4.56 \mathrm{~g}, 20 \mathrm{mmol}), 4-(\mathrm{N}, \mathrm{N}$-dimethylamino)pyridine (DMAP) (0.4g, $2 \mathrm{mmol})$ and dry chloroform (25ml) was stirred for 10 minutes. To this mixture, N, N'dicyclohexylcarbodiimide (DCC) (4.12 g, $22 \mathrm{mmol}$ ) was added and the stirring continued for 10 hours at room temperature. The precipitated $\mathrm{N}, \mathrm{N}^{\prime}$-dicyclohexylurea was filtered off and washed with excess of chloroform $(100 \mathrm{ml})$. The combined organic solution was washed with $5 \%$ aqueous acetic acid $(2 \times 50 \mathrm{ml})$, cold $5 \%$ aqueous sodium hydroxide $(2 \times$ $50 \mathrm{ml})$ and finally washed with water $(3 \times 50 \mathrm{ml})$ and dried over anhydrous sodium sulphate. The solvent was removed and the solid material so obtained was purified by column chromatography on silica gel using $1 \%$ ethyl acetate in chloroform as an eluent. Removal of solvent from the eluate afforded a white solid. This was crystallized from a mixture of chloroform and acetonitrile. Yield: $4.8 \mathrm{~g}(81 \%)$. m.p. $217.5-218.5^{\circ} \mathrm{C} . v_{\max }$ : $2920,2860,1730,1605,1580,1500,1460,1280,1120 \mathrm{~cm}^{-1} ; \delta_{\mathrm{H}}\left(\mathrm{CDCl}_{3}, 400 \mathrm{MHz}\right)$ : 8.21-8.19(d, 4H, $\left.{ }^{3} J 8.84 \mathrm{~Hz}, \mathrm{Ar}-\mathrm{H}\right), 7.92-7.90\left(\mathrm{~d}, 2 \mathrm{H},{ }^{3} J 8.0 \mathrm{~Hz}, \mathrm{Ar}-\mathrm{H}\right), 7.655-7.650(\mathrm{~d}, 2 \mathrm{H}$, $\left.{ }^{4} J 1.96 \mathrm{~Hz}, \mathrm{Ar}-\mathrm{H}\right), 7.46-7.33(\mathrm{~m}, 12 \mathrm{H}, \mathrm{Ar}-\mathrm{H}), 7.09-7.07\left(\mathrm{~d}, 4 \mathrm{H},{ }^{3} J 8.84 \mathrm{~Hz}, \mathrm{Ar}-\mathrm{H}\right) .5 .18(\mathrm{~s}$, 
$\left.4 \mathrm{H}, 2 \times \mathrm{ArCH}_{2} \mathrm{O}-\right)$. Elemental analysis for $\mathrm{C}_{38} \mathrm{H}_{28} \mathrm{O}_{6}$ : requires $\mathrm{C} 78.60, \mathrm{H} \mathrm{4.86 \%}$; found $\mathrm{C}$ $78.52, \mathrm{H} 4.59 \%$.

2,7-Naphthylene bis(4-hydroxybenzoate), (4, synthetic scheme)

2,7-Naphthylene bis (4-benzyloxybenzoate) (3) (4.5 g, $11.25 \mathrm{mmol}$ ) was dissolved in 1,4dioxane $(50 \mathrm{ml})$ and $5 \% \mathrm{Pd}-\mathrm{C}$ catalyst $(1.2 \mathrm{~g})$ was added to it. The mixture was stirred at $50^{\circ} \mathrm{C}$ in an atmosphere of hydrogen till the required quantity of hydrogen was absorbed. The resultant mixture was filtered in hot condition and the solvent removed under reduced pressure. The solid material so obtained was passed through a column of silica gel and eluted using a mixture of 5\% acetone in chloroform. Removal of solvent from the eluate gave a white material, which was crystallized using a mixture of 1,4-dioxane and petroleum-ether (b.p. $60-80^{\circ} \mathrm{C}$ ). Yield: $3.0 \mathrm{~g}(89 \%)$ m.p. $>250^{\circ} \mathrm{C}$ (d). $v_{\max }: 3330,1720$, 1605, 1280, $1120 \mathrm{~cm}^{-1} ; \delta_{\mathrm{H}}\left(\mathrm{CDCl}_{3}, 400 \mathrm{MHz}\right): 9.73(\mathrm{~s}, 2 \mathrm{H}, 2 \times \mathrm{Ar}-\mathrm{OH}$, exchangeable with $\left.\mathrm{D}_{2} \mathrm{O}\right), 8.26-8.17(\mathrm{~m}, 6 \mathrm{H}, \mathrm{Ar}-\mathrm{H}), 7.94-7.93\left(\mathrm{~d}, 2 \mathrm{H},{ }^{4} J 2.2 \mathrm{~Hz}, \mathrm{Ar}-\mathrm{H}\right), 7.59-7.56(\mathrm{~m}, 2 \mathrm{H}$, Ar-H), 7.18-7.14 (m, 4H, Ar-H). Elemental analysis for $\mathrm{C}_{24} \mathrm{H}_{16} 0_{6}$ : requires $\mathrm{C} 72.06, \mathrm{H}$ 4.03\%; found C 72.43, H 3.97\%.

\section{2,7-Naphthylene bis[4-(3-fluoro-4-n-butyloxybenzoyloxy)benzoate], (10,}

\section{Compound No.)}

This and other homologues were synthesized following a procedure described for the preparation of compound (3).

Yield: $67 \%$. m.p. $170.0^{\circ}$ C. $v_{\max }: 2924,2854,1736,1601,1462,1267,1132 \mathrm{~cm}^{-1}$; $\delta_{\mathrm{H}}\left(\mathrm{CDCl}_{3}, 400 \mathrm{MHz}\right): 8.34-8.32\left(\mathrm{~d}, 4 \mathrm{H},{ }^{3} J 8.72 \mathrm{~Hz}, \mathrm{Ar}-\mathrm{H}\right), 7.99-7.9(\mathrm{~m}, 6 \mathrm{H}, \mathrm{Ar}-\mathrm{H}), 7.709-$ 7.704(d, 2H, $\left.{ }^{4} J 2.04 \mathrm{~Hz}, \mathrm{Ar}-\mathrm{H}\right), 7.40-7.37(\mathrm{~m}, 6 \mathrm{H}, \mathrm{Ar}-\mathrm{H}), 7.08-7.03\left(\mathrm{t}, 2 \mathrm{H},{ }^{3} J 8.24 \mathrm{~Hz}, \mathrm{Ar}-\right.$ 
H), 4.17-4.13(t, $4 \mathrm{H},{ }^{3} J 6.48 \mathrm{~Hz}, 2 \times \mathrm{Ar}-\mathrm{OCH}_{2}-\mathrm{CH}_{2}-$ ), $1.9-1.83$ (quin, $4 \mathrm{H},{ }^{3} J 6.52 \mathrm{~Hz}, 2 \times$ Ar- $\left.-\mathrm{OCH}_{2}-\mathrm{CH}_{2}-\mathrm{CH}_{2}-\right), 1.59-1.50\left(\mathrm{~m}, 4 \mathrm{H}, 2 \times-\mathrm{CH}_{2}-\mathrm{CH}_{2}-\mathrm{CH}_{3}\right), 1.03-0.99\left(\mathrm{t}, 6 \mathrm{H},{ }^{3} J 7.36 \mathrm{~Hz}\right.$, $\left.2 \times-\mathrm{CH}_{2}-\underline{\mathrm{CH}_{3}}\right)$.

\section{2,7-Naphthylene bis[4-(3-fluoro-4-n-heptyloxybenzoyloxy)benzoate], (11)}

Yield: $69 \%$. m.p. $164.0^{\circ} \mathrm{C} . v_{\max }: 2926,2854,1736,1728,1618,1460,1283,1136 \mathrm{~cm}^{-1} ; \delta_{\mathrm{H}}$ (CDCl $3,400 \mathrm{MHz}):$ 8.34-8.32(d, 4H, $\left.{ }^{3} J 8.64 \mathrm{~Hz}, \mathrm{Ar}-\mathrm{H}\right), 7.99-7.9(\mathrm{~m}, 6 \mathrm{H}, \mathrm{Ar}-\mathrm{H}), 7.709-$ 7.704(d, $\left.2 \mathrm{H},{ }^{4} J 1.84 \mathrm{~Hz}, \mathrm{Ar}-\mathrm{H}\right), 7.41-7.37(\mathrm{~m}, 6 \mathrm{H}, \mathrm{Ar}-\mathrm{H}), 7.07-7.03\left(\mathrm{t}, 2 \mathrm{H},{ }^{3} J 8.28 \mathrm{~Hz}, \mathrm{Ar}-\right.$ $\mathrm{H}), 4.16-4.12\left(\mathrm{t}, 4 \mathrm{H},{ }^{3} \mathrm{~J} 6.52 \mathrm{~Hz}, 2 \times \mathrm{Ar}-\mathrm{OCH}_{2}-\mathrm{CH}_{2}\right.$ ), $1.91-1.84$ (quin, $4 \mathrm{H},{ }^{3} J 6.6 \mathrm{~Hz}, 2 \times$ Ar- $\mathrm{OCH}_{2}-\mathrm{CH}_{2}-\mathrm{CH}_{2}-$ ), $1.50-1.47$ (quin, $4 \mathrm{H},{ }^{3} \mathrm{~J} 7.92 \mathrm{~Hz}, 2 \times-\mathrm{CH}_{2}-\mathrm{CH}_{2}-\mathrm{CH}_{2}-$ ), 1.41-1.33(m, $\left.12 \mathrm{H}, 6 \times-\mathrm{CH}_{2}-\mathrm{CH}_{2}-\mathrm{CH}_{2}-\right), 0.93-0.89\left(\mathrm{t}, 6 \mathrm{H},{ }^{3} J 6.64 \mathrm{~Hz}, 2 \times-\mathrm{CH}_{2}-\mathrm{CH}_{3}\right)$.

\section{2,7-Naphthylene bis[4-(3-fluoro-4-n-nonyloxybenzoyloxy)benzoate]，(12)}

Yield: $66 \%$ m.p. $156.0^{\circ} \mathrm{C}$. $v_{\max }: 2924,2852,1736,1726,1618,1460,1283,1140 \mathrm{~cm}^{-1}$; $\delta_{\mathrm{H}}\left(\mathrm{CDCl}_{3}, 400 \mathrm{MHz}\right): 8.34-8.32\left(\mathrm{~d}, 4 \mathrm{H},{ }^{3} J 8.64 \mathrm{~Hz}, \mathrm{Ar}-\mathrm{H}\right), 7.99-7.9(\mathrm{~m}, 6 \mathrm{H}, \mathrm{Ar}-\mathrm{H}), 7.709-$ 7.705(d, 2H, $\left.{ }^{4} J 1.56 \mathrm{~Hz}, \mathrm{Ar}-\mathrm{H}\right), 7.41-7.37(\mathrm{~m}, 6 \mathrm{H}, \mathrm{Ar}-\mathrm{H}), 7.07-7.03\left(\mathrm{t}, 2 \mathrm{H},{ }^{3} \mathrm{~J} 8.28 \mathrm{~Hz}, \mathrm{Ar}-\right.$ H), 4.16-4.12(t, 4H, ${ }^{3} J 6.52 \mathrm{~Hz}, 2 \times \mathrm{Ar}-\mathrm{OCH}_{2}-\mathrm{CH}_{2}$ ) $)$ 1.91-1.84(quin, $4 \mathrm{H},{ }^{3} J 6.68 \mathrm{~Hz}, 2 \times$ Ar- $-\mathrm{OCH}_{2}-\mathrm{CH}_{2}-\mathrm{CH}_{2}$ ), $1.52-1.47$ (quin, $\left.4 \mathrm{H},{ }^{3} J 7.2 \mathrm{~Hz}, 2 \times-\mathrm{CH}_{2}-\underline{\mathrm{CH}}_{2}-\mathrm{CH}_{2}-\right), 1.38-1.3(\mathrm{~m}$, $\left.20 \mathrm{H}, 10 \times-\mathrm{CH}_{2}-\underline{\mathrm{CH}}_{2}-\mathrm{CH}_{2}-\right), 0.91-0.88\left(\mathrm{t}, 6 \mathrm{H},{ }^{3} \mathrm{~J} 6.24 \mathrm{~Hz}, 2 \times-\mathrm{CH}_{2}-\underline{\mathrm{CH}}_{3}\right)$.

\section{2,7-Naphthylene bis[4-(3-fluoro-4-n-decyloxybenzoyloxy)benzoate], (13)}

Yield: $68 \%$, m.p. $156.5^{\circ}$ C. $v_{\max }: 2924,2853,1736,1728,1618,1458,1283,1140 \mathrm{~cm}^{-1}$; $\delta_{\mathrm{H}}\left(\mathrm{CDCl}_{3}, 400 \mathrm{MHz}\right): 8.34-8.32\left(\mathrm{~d}, 4 \mathrm{H},{ }^{3} J 8.68 \mathrm{~Hz}, \mathrm{Ar}-\mathrm{H}\right), 7.99-7.9(\mathrm{~m}, 6 \mathrm{H}, \mathrm{Ar}-\mathrm{H}), 7.71-$ 7.705(d, 2H, $\left.{ }^{4} J 1.88 \mathrm{~Hz}, \mathrm{Ar}-\mathrm{H}\right), 7.41-7.37(\mathrm{~m}, 6 \mathrm{H}, \mathrm{Ar}-\mathrm{H}), 7.07-7.03\left(\mathrm{t}, 2 \mathrm{H},{ }^{3} J 8.28 \mathrm{~Hz}, \mathrm{Ar}-\right.$ 
$\mathrm{H}), 4.16-4.12\left(\mathrm{t}, 4 \mathrm{H},{ }^{3} \mathrm{~J} 6.56 \mathrm{~Hz}, 2 \times \mathrm{Ar}-\mathrm{OCH} \underline{\mathrm{CH}}_{2}-\mathrm{CH}_{2}-\right)$ ) $1.91-1.84$ (quin, $4 \mathrm{H},{ }^{3} J 6.64 \mathrm{~Hz}, 2 \times$ Ar- $-\mathrm{OCH}_{2}-\underline{\mathrm{CH}}_{2}-\mathrm{CH}_{2}$ ), $1.50-1.46\left(\right.$ quin, $4 \mathrm{H},{ }^{3} \mathrm{~J} 7.12 \mathrm{~Hz}, 2 \times-\mathrm{CH}_{2}-\mathrm{CH}_{2}-\mathrm{CH}_{2}$ ), $1.38-1.29(\mathrm{~m}$, $\left.24 \mathrm{H}, 12 \times-\mathrm{CH}_{2}-\underline{\mathrm{CH}_{2}}-\mathrm{CH}_{2}-\right), 0.91-0.87\left(\mathrm{t}, 6 \mathrm{H},{ }^{3} \mathrm{~J} 6.44 \mathrm{~Hz}, 2 \times-\mathrm{CH}_{2}-\underline{\mathrm{CH}}_{3}\right)$.

\section{2,7-Naphthylene bis[4-(3-fluoro-4-n-undecyloxybenzoyloxy)benzoate], (14)}

Yield: $69 \%$. m.p. $154.5^{\circ}$ C. $v_{\max }: 2916,2851,1736,1728,1618,1458,1283,1142 \mathrm{~cm}^{-1}$; $\delta_{\mathrm{H}}\left(\mathrm{CDCl}_{3}, 400 \mathrm{MHz}\right): 8.35-8.32\left(\mathrm{~d}, 4 \mathrm{H},{ }^{3} J 8.64 \mathrm{~Hz}, \mathrm{Ar}-\mathrm{H}\right), 7.99-7.9(\mathrm{~m}, 6 \mathrm{H}, \mathrm{Ar}-\mathrm{H}), 7.709-$ 7.705(d, 2H, $\left.{ }^{4} J 1.8 \mathrm{~Hz}, \mathrm{Ar}-\mathrm{H}\right), 7.41-7.38(\mathrm{~m}, 6 \mathrm{H}, \mathrm{Ar}-\mathrm{H}), 7.07-7.03\left(\mathrm{t}, 2 \mathrm{H},{ }^{3} J 8.24 \mathrm{~Hz}, \mathrm{Ar}-\right.$ H), 4.15-4.12(t, 4H, ${ }^{3} J 6.52 \mathrm{~Hz}, 2 \times \mathrm{Ar}-\mathrm{OCH} \underline{2}_{2}-\mathrm{CH}_{2}$ ) $), 1.91-1.84$ (quin, $4 \mathrm{H},{ }^{3} J 6.64 \mathrm{~Hz}, 2 \times$ $\mathrm{Ar}-\mathrm{OCH}{ }_{2}-\underline{\mathrm{CH}}_{2}-\mathrm{CH}_{2}$ ) $), 1.52-1.46$ (quin, $\left.4 \mathrm{H},{ }^{3} \mathrm{J7} .2 \mathrm{~Hz}, 2 \times-\mathrm{CH}_{2}-\underline{\mathrm{CH}_{2}}-\mathrm{CH}_{2}-\right), 1.38-1.28(\mathrm{~m}$, $\left.28 \mathrm{H}, 14 \times-\mathrm{CH}_{2}-\underline{\mathrm{CH}}_{2}-\mathrm{CH}_{2}-\right), 0.91-0.87\left(\mathrm{t}, 6 \mathrm{H},{ }^{3} J 6.4 \mathrm{~Hz}, 2 \times-\mathrm{CH}_{2}-\mathrm{CH}_{3}\right)$.

2,7-Naphthylene bis[4-(3-fluoro-4-n-dodecyloxybenzoyloxy)benzoate], (15)

Yield: $72 \%$. m.p. $155.0^{\circ}$ C. $v_{\max }: 2916,2849,1736,1728,1608,1458,1283,1142 \mathrm{~cm}^{-1}$; $\delta_{\mathrm{H}}\left(\mathrm{CDCl}_{3}, 400 \mathrm{MHz}\right): 8.35-8.32\left(\mathrm{~d}, 4 \mathrm{H},{ }^{3} J 8.72 \mathrm{~Hz}, \mathrm{Ar}-\mathrm{H}\right), 7.99-7.9(\mathrm{~m}, 6 \mathrm{H}, \mathrm{Ar}-\mathrm{H}), 7.71-$ 7.705(d, 2H, $\left.{ }^{4} J 1.92 \mathrm{~Hz}, \mathrm{Ar}-\mathrm{H}\right), 7.41-7.37(\mathrm{~m}, 6 \mathrm{H}, \mathrm{Ar}-\mathrm{H}), 7.07-7.03\left(\mathrm{t}, 2 \mathrm{H},{ }^{3} J 8.28 \mathrm{~Hz}, \mathrm{Ar}-\right.$ $\mathrm{H}), 4.15-4.12\left(\mathrm{t}, 4 \mathrm{H},{ }^{3} \mathrm{~J} 6.52 \mathrm{~Hz}, 2 \times \mathrm{Ar}-\mathrm{OCH}_{2}-\mathrm{CH}_{2}\right.$ ), $1.91-1.84$ (quin, $4 \mathrm{H},{ }^{3} J 6.6 \mathrm{~Hz}, 2 \times$ Ar- $\mathrm{OCH}_{2}-\mathrm{CH}_{2}-\mathrm{CH}_{2}-$ ), 1.52-1.46(quin, $4 \mathrm{H},{ }^{3} J 7.32 \mathrm{~Hz}, 2 \times-\mathrm{CH}_{2}-\mathrm{CH}_{2}-\mathrm{CH}_{2}-$ ), 1.38-1.28(m, $\left.32 \mathrm{H}, 16 \times-\mathrm{CH}_{2}-\mathrm{CH}_{2}-\mathrm{CH}_{2}-\right), 0.91-0.87\left(\mathrm{t}, 6 \mathrm{H},{ }^{3} \mathrm{~J} 6.52 \mathrm{~Hz}, 2 \times-\mathrm{CH}_{2}-\underline{\mathrm{CH}_{3}}\right)$.

\section{2,7-Naphthylene bis[4-(3-fluoro-4-n-tridecyloxybenzoyloxy)benzoate], (16)}

Yield: $68 \%$. m.p. $155.0^{\circ}$ C. $v_{\max }: 2916,2849,1736,1728,1608,1458,1283,1142 \mathrm{~cm}^{-1}$; $\delta_{\mathrm{H}}\left(\mathrm{CDCl}_{3}, 400 \mathrm{MHz}\right): 8.35-8.32\left(\mathrm{~d}, 4 \mathrm{H},{ }^{3} J 8.72 \mathrm{~Hz}, \mathrm{Ar}-\mathrm{H}\right), 7.99-7.9(\mathrm{~m}, 6 \mathrm{H}, \mathrm{Ar}-\mathrm{H}), 7.71-$ 7.705(d, 2H, $\left.{ }^{4} J 1.92 \mathrm{~Hz}, \mathrm{Ar}-\mathrm{H}\right), 7.41-7.37(\mathrm{~m}, 6 \mathrm{H}, \mathrm{Ar}-\mathrm{H}), 7.07-7.03\left(\mathrm{t}, 2 \mathrm{H},{ }^{3} \mathrm{~J} 8.28 \mathrm{~Hz}, \mathrm{Ar}-\right.$ 
$\mathrm{H}), 4.15-4.12\left(\mathrm{t}, 4 \mathrm{H},{ }^{3} \mathrm{~J} 6.52 \mathrm{~Hz}, 2 \times \mathrm{Ar}-\mathrm{OCH}_{2}-\mathrm{CH}_{2}-\right.$ ), $1.91-1.84$ (quin, $4 \mathrm{H},{ }^{3} \mathrm{~J} 6.6 \mathrm{~Hz}, 2 \times$ Ar- $\mathrm{OCH}_{2}-\mathrm{CH}_{2}-\mathrm{CH}_{2}-$ ), 1.52-1.46(quin, $4 \mathrm{H},{ }^{3} J 7.32 \mathrm{~Hz}, 2 \times-\mathrm{CH}_{2}-\mathrm{CH}_{2}-\mathrm{CH}_{2}-$ ), 1.38-1.28(m, $\left.36 \mathrm{H}, 18 \times-\mathrm{CH}_{2}-\underline{\mathrm{CH}}_{2}-\mathrm{CH}_{2}-\right), 0.91-0.87\left(\mathrm{t}, 6 \mathrm{H},{ }^{3} \mathrm{~J} 6.52 \mathrm{~Hz}, 2 \times-\mathrm{CH}_{2}-\underline{\mathrm{CH}}_{3}\right)$.

\section{2,7-Naphthylene bis[4-(3-fluoro-4-n-tetradecyloxybenzoyloxy)benzoate], (17)}

Yield: $68 \%$. m.p. $155.5^{\circ}$ C. $v_{\max }: 2916,2849,1736,1728,1618,1458,1283,1142 \mathrm{~cm}^{-1}$; $\delta_{\mathrm{H}}\left(\mathrm{CDCl}_{3}, 400 \mathrm{MHz}\right): 8.35-8.32\left(\mathrm{~d}, 4 \mathrm{H},{ }^{3} J 8.64 \mathrm{~Hz}, \mathrm{Ar}-\mathrm{H}\right), 7.99-7.9(\mathrm{~m}, 6 \mathrm{H}, \mathrm{Ar}-\mathrm{H}), 7.71-$ 7.705(d, 2H, $\left.{ }^{4} J 1.6 \mathrm{~Hz}, \mathrm{Ar}-\mathrm{H}\right), 7.41-7.37(\mathrm{~m}, 6 \mathrm{H}, \mathrm{Ar}-\mathrm{H}), 7.07-7.03\left(\mathrm{t}, 2 \mathrm{H},{ }^{3} J 8.32 \mathrm{~Hz}, \mathrm{Ar}-\right.$ H), 4.15-4.12(t, 4H, ${ }^{3} J 6.52 \mathrm{~Hz}, 2 \times \mathrm{Ar}-\mathrm{OCH} \underline{2}_{2}-\mathrm{CH}_{2}$ ) $), 1.91-1.84$ (quin, $4 \mathrm{H},{ }^{3} J 6.64 \mathrm{~Hz}, 2 \times$ Ar- $\mathrm{OCH}_{2}-\underline{\mathrm{CH}}_{2}-\mathrm{CH}_{2}$ ) $), 1.50-1.46$ (quin, $4 \mathrm{H},{ }^{3} \mathrm{~J} 7.16 \mathrm{~Hz}, 2 \times-\mathrm{CH}_{2}-\mathrm{CH}_{2}-\mathrm{CH}_{2}-$ ), 1.38-1.27(m, $\left.40 \mathrm{H}, 20 \times-\mathrm{CH}_{2}-\underline{\mathrm{CH}}_{2}-\mathrm{CH}_{2}-\right), 0.9-0.87\left(\mathrm{t}, 6 \mathrm{H},{ }^{3} J 6.32 \mathrm{~Hz}, 2 \times-\mathrm{CH}_{2}-\mathrm{CH}_{3}\right)$.

\section{2,7-Naphthylene bis[4-(3-fluoro-4-n-pentadecyloxybenzoyloxy)benzoate], (18)}

Yield: $65 \%$. m.p. $152.5^{\circ}$ C. $v_{\max }$ : 2916, 2849, 1736, 1728, 1618, 1458, 1283, $1142 \mathrm{~cm}^{-1}$; $\delta_{\mathrm{H}}\left(\mathrm{CDCl}_{3}, 400 \mathrm{MHz}\right): 8.35-8.32\left(\mathrm{~d}, 4 \mathrm{H},{ }^{3} J 8.64 \mathrm{~Hz}, \mathrm{Ar}-\mathrm{H}\right), 7.99-7.9(\mathrm{~m}, 6 \mathrm{H}, \mathrm{Ar}-\mathrm{H}), 7.71-$ 7.705(d, 2H, $\left.{ }^{4} J 1.6 \mathrm{~Hz}, \mathrm{Ar}-\mathrm{H}\right), 7.41-7.37(\mathrm{~m}, 6 \mathrm{H}, \mathrm{Ar}-\mathrm{H}), 7.07-7.03\left(\mathrm{t}, 2 \mathrm{H},{ }^{3} J 8.32 \mathrm{~Hz}, \mathrm{Ar}-\right.$ $\mathrm{H}), 4.15-4.12\left(\mathrm{t}, 4 \mathrm{H},{ }^{3} J 6.52 \mathrm{~Hz}, 2 \times \mathrm{Ar}-\mathrm{OCH}_{2}-\mathrm{CH}_{2}\right.$ ) $)$ 1.91-1.84(quin, $4 \mathrm{H},{ }^{3} J 6.64 \mathrm{~Hz}, 2 \times$ Ar- $\mathrm{OCH}_{2}-\mathrm{CH}_{2}-\mathrm{CH}_{2}-$ ), 1.50-1.46(quin, $4 \mathrm{H},{ }^{3} J 7.16 \mathrm{~Hz}, 2 \times-\mathrm{CH}_{2}-\mathrm{CH}_{2}-\mathrm{CH}_{2}-$ ), 1.38-1.27(m, $\left.44 \mathrm{H}, 22 \times-\mathrm{CH}_{2}-\mathrm{CH}_{2}-\mathrm{CH}_{2}-\right), 0.9-0.87\left(\mathrm{t}, 6 \mathrm{H},{ }^{3} J 6.32 \mathrm{~Hz}, 2 \times-\mathrm{CH}_{2}-\mathrm{CH}_{3}\right)$.

\section{2,7-Naphthylene bis[4-(3-fluoro-4-n-hexadecyloxybenzoyloxy)benzoate],}

Yield: $71 \%$. m.p. $152.5^{\circ}$ C. $v_{\max }: 2916,2849,1736,1728,1618,1470,1284,1142 \mathrm{~cm}^{-1}$; $\delta_{\mathrm{H}}\left(\mathrm{CDCl}_{3}, 400 \mathrm{MHz}\right): 8.35-8.32\left(\mathrm{~d}, 4 \mathrm{H},{ }^{3} J 8.68 \mathrm{~Hz}, \mathrm{Ar}-\mathrm{H}\right), 7.99-7.9(\mathrm{~m}, 6 \mathrm{H}, \mathrm{Ar}-\mathrm{H}), 7.709-$ 7.706(d, 2H, $\left.{ }^{4} J 1.32 \mathrm{~Hz}, \mathrm{Ar}-\mathrm{H}\right), 7.41-7.37(\mathrm{~m}, 6 \mathrm{H}, \mathrm{Ar}-\mathrm{H})$, 7.07-7.03(t, $2 \mathrm{H},{ }^{3} \mathrm{~J} 8.32 \mathrm{~Hz}, \mathrm{Ar}-$ 
$\mathrm{H}), 4.15-4.12\left(\mathrm{t}, 4 \mathrm{H},{ }^{3} \mathrm{~J} 6.52 \mathrm{~Hz}, 2 \times \mathrm{Ar}-\mathrm{OCH} \underline{\mathrm{CH}}_{2}-\mathrm{CH}_{2}-\right.$ ), $1.91-1.84$ (quin, $4 \mathrm{H},{ }^{3} J 6.68 \mathrm{~Hz}, 2 \times$ Ar- $\mathrm{OCH}_{2}-\mathrm{CH}_{2}-\mathrm{CH}_{2}-$ ), 1.52-1.46(quin, $4 \mathrm{H},{ }^{3} J 7.28 \mathrm{~Hz}, 2 \times-\mathrm{CH}_{2}-\mathrm{CH}_{2}-\mathrm{CH}_{2}-$ ), 1.38-1.27(m, $\left.48 \mathrm{H}, 24 \times-\mathrm{CH}_{2}-\underline{\mathrm{CH}}_{2}-\mathrm{CH}_{2}-\right), 0.9-0.87\left(\mathrm{t}, 6 \mathrm{H},{ }^{3} J 6.32 \mathrm{~Hz}, 2 \times-\mathrm{CH}_{2}-\mathrm{CH}_{3}\right)$.

\section{2,7-Naphthylene bis[4-(3-fluoro-4-n-octadecyloxybenzoyloxy)benzoate], (20)}

Yield: $69 \%$. m.p. $151.0^{\circ} \mathrm{C} . v_{\max }: 2916,2849,1736,1728,1618,1470,1284,1142 \mathrm{~cm}^{-1}$; $\delta_{\mathrm{H}}\left(\mathrm{CDCl}_{3}, 400 \mathrm{MHz}\right): 8.35-8.32\left(\mathrm{~d}, 4 \mathrm{H},{ }^{3} J 8.64 \mathrm{~Hz}, \mathrm{Ar}-\mathrm{H}\right), 7.99-7.9(\mathrm{~m}, 6 \mathrm{H}, \mathrm{Ar}-\mathrm{H}), 7.709-$ 7.706(d, 2H, $\left.{ }^{4} J 1.6 \mathrm{~Hz}, \mathrm{Ar}-\mathrm{H}\right), 7.41-7.37(\mathrm{~m}, 6 \mathrm{H}, \mathrm{Ar}-\mathrm{H}), 7.07-7.03\left(\mathrm{t}, 2 \mathrm{H},{ }^{3} J 8.32 \mathrm{~Hz}, \mathrm{Ar}-\right.$ H), 4.15-4.12(t, 4H, ${ }^{3} J 6.52 \mathrm{~Hz}, 2 \times \mathrm{Ar}-\mathrm{OCH}_{2}-\mathrm{CH}_{2}$ ) $)$ 1.91-1.84(quin, $4 \mathrm{H},{ }^{3} J 6.68 \mathrm{~Hz}, 2 \times$ $\mathrm{Ar}-\mathrm{OCH}{ }_{2}-\underline{\mathrm{CH}}_{2}-\mathrm{CH}_{2}$ ) $)$ 1.52-1.46(quin, $\left.4 \mathrm{H},{ }^{3} J 7.26 \mathrm{~Hz}, 2 \times-\mathrm{CH}_{2}-\underline{\mathrm{CH}_{2}}-\mathrm{CH}_{2}-\right), 1.38-1.27(\mathrm{~m}$, $\left.56 \mathrm{H}, 28 \times-\mathrm{CH}_{2}-\underline{\mathrm{CH}}_{2}-\mathrm{CH}_{2}-\right), 0.9-0.87\left(\mathrm{t}, 6 \mathrm{H},{ }^{3} \mathrm{~J} 6.3 \mathrm{~Hz}, 2 \times-\mathrm{CH}_{2}-\underline{\mathrm{CH}_{3}}\right)$.

2,7-Naphthylene bis[4-(3-fluoro-4-n-eicocyloxybenzoyloxy)benzoate]，(21)

Yield: $68 \%$. m.p. $149.5^{\circ}$ C. $v_{\max }: 2916,2849,1734,1728,1618,1458,1284,1140 \mathrm{~cm}^{-1}$; $\delta_{\mathrm{H}}\left(\mathrm{CDCl}_{3}, 400 \mathrm{MHz}\right): 8.35-8.33\left(\mathrm{~d}, 4 \mathrm{H},{ }^{3} J 8.4 \mathrm{~Hz}, \mathrm{Ar}-\mathrm{H}\right), 7.99-7.9(\mathrm{~m}, 6 \mathrm{H}, \mathrm{Ar}-\mathrm{H})$, 7.7097.706(d, 2H, $\left.{ }^{4} J 1.8 \mathrm{~Hz}, \mathrm{Ar}-\mathrm{H}\right), 7.41-7.39(\mathrm{~m}, 6 \mathrm{H}, \mathrm{Ar}-\mathrm{H}), 7.07-7.03\left(\mathrm{t}, 2 \mathrm{H},{ }^{3} J 8.32 \mathrm{~Hz}, \mathrm{Ar}-\right.$ H), 4.15-4.12(t, 4H, ${ }^{3} J 6.28 \mathrm{~Hz}, 2 \times \mathrm{Ar}-\mathrm{OCH}_{2}-\mathrm{CH}_{2}$ ) $), 1.89-1.84$ (quin, $4 \mathrm{H},{ }^{3} J 6.96 \mathrm{~Hz}, 2 \times$ Ar- $\mathrm{OCH}_{2}-\underline{\mathrm{CH}}_{2}-\mathrm{CH}_{2}-$ ), $1.53-1.5\left(\right.$ quin, $\left.4 \mathrm{H},{ }^{3} J 7.2 \mathrm{~Hz}, 2 \times-\mathrm{CH}_{2}-\mathrm{CH}_{2}-\mathrm{CH}_{2}-\right)$, 1.38-1.25(m, $\left.64 \mathrm{H}, 32 \times-\mathrm{CH}_{2}-\mathrm{CH}_{2}-\mathrm{CH}_{2}-\right), 0.9-0.86\left(\mathrm{t}, 6 \mathrm{H},{ }^{3} J 6.92 \mathrm{~Hz}, 2 \times-\mathrm{CH}_{2}-\underline{\mathrm{CH}_{3}}\right)$. 